\title{
All-Solid-State Proton-Based Tandem Structure Achieving Ultrafast Switching Electrochromic Windows
}

\section{Zewei Shao}

Shanghai institute of Ceramics, Chinese Academy of Sciences

\section{Aibin huang}

Shanghai institute of Ceramics, Chinese Academy of Sciences

Chen Ming

Shanghai Institute of Ceramics

John Bell

The Queensland University of Technology

Pu Yu

Tsinghua University https://orcid.org/0000-0002-5513-7632

\section{Yi-Yang Sun}

Shanghai Institute of Ceramics

Liangmao Jin

State Key Laboratory of Advanced Technology for Float Glass

\section{Liyun Ma}

State Key Laboratory of Advanced Technology for Float Glass

Hongjie Luo

Shanghai University

\section{Ping Jin}

Shanghai institute of Ceramics, Chinese Academy of Sciences

\section{Xun Cao ( $\nabla$ cxun@mail.sic.ac.cn )}

Shanghai institute of Ceramics, Chinese Academy of Sciences

\section{Article}

Keywords: all-solid-state electrochromic devices (ECDs), smart-window applications

Posted Date: April 14th, 2021

DOI: https://doi.org/10.21203/rs.3.rs-321790/v1 
License: (c) (i) This work is licensed under a Creative Commons Attribution 4.0 International License. Read Full License

Version of Record: A version of this preprint was published at Nature Electronics on January 24th, 2022. See the published version at https://doi.org/10.1038/s41928-021-00697-4. 


\section{All-Solid-State Proton-Based Tandem Structure Achieving Ultrafast Switching Electrochromic Windows}

Zewei Shao ${ }^{1,2, \#}$, Aibin Huang ${ }^{1,2, \#, ~ C h e n ~ M i n g ~}{ }^{1,2, \#}$, John Bell ${ }^{3}$, Pu Yu ${ }^{4}$, Yi-Yang Sun ${ }^{1,2, *}$, Liangmao Jin ${ }^{5}$, Liyun $\mathrm{Ma}^{5}$, Hongjie Luo ${ }^{6, *}$, Ping $\mathrm{Jin}^{1,2,7}$ and Xun $\mathrm{Cao}^{1,2,{ }^{*}}$

${ }^{1}$ State Key Laboratory of High Performance Ceramics and Superfine Microstructure, Shanghai Institute of Ceramics, Chinese Academy of Sciences, Shanghai 201899, China

${ }^{2}$ University of Chinese Academy of Sciences, Beijing 100049, China

${ }^{3}$ Chemistry, Physics and Mechanical Engineering Science and Engineering Faculty,

Queensland University of Technology, Brisbane, Queensland 4001, Australia

${ }^{4}$ State Key Laboratory of Low Dimensional Quantum Physics and Department of Physics, Tsinghua University, Beijing 100084, China.

${ }^{5}$ State Key Laboratory of Advanced Technology for Float Glass, Bengbu, Anhui 233000, China

${ }^{6}$ School of Materials Science and Engineering, Shanghai University, Shanghai 200444, China

${ }^{7}$ Materials Research Institute for Sustainable Development, National Institute of Advanced Industrial Science and Technology, Nagoya 463-8560, Japan

\section{Summary of Manuscript:}

Sub-second coloration of electrochromic window is realized by introducing a solid-state proton source and a tandem structure. 


\begin{abstract}
All-solid-state electrochromic devices (ECDs) for smart-window applications currently suffer from limited ion diffusion speed, which lead to slow coloration and bleaching processes. Here, we design an all-solid-state tandem structure with protons as diffusing species achieving an ultrafast switching ECD. We use $\mathrm{WO}_{3}$ as the electrochromic material, while poly(3,4-ethylenedioxythiophene): polystyrene sulfonate (PEDOT:PSS) as the solid-state proton source to enable fast switching. This structure by itself exhibits low optical modulation (i.e., difference of on/off transmittance). We further introduce a solid polymeric electrolyte layer on top of PEDOT:PSS to form a tandem structure, which provides $\mathrm{Na}^{+}$ions to PEDOT:PSS and pump protons there to the $\mathrm{WO}_{3}$ layer through ion exchange. Our new all-solid-state ECD features high optical modulation (>92\% at $650 \mathrm{~nm}$ ), fast response (coloration to $90 \%$ in $0.7 \mathrm{~s}$ and bleaching to $65 \%$ in $0.9 \mathrm{~s}$ and $90 \%$ in $7.1 \mathrm{~s}$ ) and excellent stability (<10\% degradation after 3000 cycles). Large-area $\left(30 \times 40 \mathrm{~cm}^{2}\right)$ as well as flexible devices are fabricated to demonstrate the great potential for scaling up.
\end{abstract}




\section{Introduction}

Electrochromic smart windows, which can regulate the transmittance of solar radiation by applying a voltage, have been regarded as a promising method for enhancing building energy efficiency. ${ }^{1-4}$ Overall consideration on production cost, coloration efficiency, response speed and cycle durability suggests that tungsten trioxide $\left(\mathrm{WO}_{3}\right)$ is the most preferred candidate for large-scale application of electrochromic devices (ECDs). ${ }^{5-7}$ Previous works suggested that when small ions such as $\mathrm{H}^{+}, \mathrm{Li}^{+}, \mathrm{Na}^{+}$or $\mathrm{Al}^{3+}$ are inserted into the $\mathrm{WO}_{3}$ lattice, transitions between small-polaron states associated with $\mathrm{W}$ ions of different valences could result in optical absorptions that are responsible for coloration of $\mathrm{WO}_{3} \cdot{ }^{8-11}$ The reversible injection and extraction of the extrinsic ions as controlled by electric field form the basic mechanism of a $\mathrm{WO}_{3}$-based ECD.

The ionic radius of the insertion ions is a critical factor determining the coloration speed of an ECD, as well as its durability because of the damage to the lattice during cycling. As shown in Fig. 1a, protons with the smallest radius and mass migrate much faster than other ions and yield higher electrochromic performance. ${ }^{12-16}$ However, in previous studies, protons are exclusively introduced by liquid electrolytes. ${ }^{15}$, 17-19 Despite the superiority of the proton-based ECDs, the use of liquid electrolyte renders them not appealing for many practical applications. ${ }^{20-22}$ Another challenging issue using protons as insertion ions is the generation of $\mathrm{H}_{2}$ gas if the applied potential is over the electrochemical potential of the $\mathrm{H}^{+} / \mathrm{H}_{2}$ pair, which incurs serious safety issue. ${ }^{23}$

Recently, studies on neuromorphic materials reveal that the organic conductive polymer poly(3,4-ethylenedioxythiophene):polystyrene sulfonate (PEDOT:PSS) can release protons under an electric field. This finding has been used to fabricate memristive devices. ${ }^{24,25}$ In the polymer mixture, PEDOT functions as an organic 
semiconductor while PSS functions as a dopant and provides hole carriers to PEDOT by removing a portion of $\mathrm{H}$ atoms from the $-\mathrm{SO}_{3} \mathrm{H}$ groups. Because of the transparent and conductive nature of PEDOT:PSS, previous studies have explored the use of PEDOT:PSS in ECDs. ${ }^{26-29}$ But in these studies, instead of a proton source PEDOT:PSS

serves as an electrode or an electrochromic layer only. ${ }^{30-33}$ A detailed comparison of these previous works has been provided in the Supporting Information (SI, Table S1).

In this paper, we design an all-solid-state tandem structure for the ECDs, which is composed of an electrochromic $\mathrm{WO}_{3}$ layer, a PEDOT:PSS layer as the proton source, and a solid polymeric electrolyte (SPE) layer on top of the PEDOT:PSS layer. This design is not merely a proof of concept using solid-state proton source in ECDs, but it also demonstrates excellent performance in coloration speed, optical modulation, and durability. As will be shown, the tandem structure manifests its multiple functions: 1) the SPE provides $\mathrm{Na}^{+}$ions to PEDOT:PSS and pumps protons to $\mathrm{WO}_{3}$ realizing a relay of the insertion ions; 2) the insulating SPE layer carries most of the applied voltage so that $\mathrm{H}_{2}$ formation in PEDOT:PSS is avoided during high-voltage operation; 3) PEDOT:PSS as an electrochromic material supplements the light absorption of $\mathrm{WO}_{3}$ and enhances the optical modulation of the overall device. Based on this design, we have fabricated large-area as well as flexible devices to demonstrate its great potential for large-scale applications.

\section{Results and discussion}

Design of the tandem-structure ECD. To achieve an all-solid-state design and use protons as insertion ions, we employed a PEDOT:PSS film as proton source. We fabricated an ECD composed of five layers: ITO/ $\mathrm{WO}_{3} / \mathrm{SiO}_{2} / \mathrm{PEDOT}: \mathrm{PSS} / \mathrm{ITO}$, where the indium tin oxide (ITO) layer serves as a transparent conducting layer and the $\mathrm{SiO}_{2}$ 
layer between the $\mathrm{WO}_{3}$ and PEDOT:PSS layers serves as an ion-conductive and electron-insulating layer. The upper panel of Fig. 1b shows the transmittance changes of this device in response to voltage pulses with the width varying from 40 to $5 / 16 \mathrm{~s}$. The wavelength of the illumination light is $670 \mathrm{~nm}$. Under voltage pulse of $40 \mathrm{~s}$, the transmittance change (i.e., the difference between the maximum and minimum transmittances, or $\Delta T$ ) is $15 \%$. Even when the pulse width is reduced to $5 / 8 \mathrm{~s}$, the device is still able to response by a $\Delta T$ of $13 \%$. When the pulse width is further reduced to $5 / 16$ $\mathrm{s}, \Delta T$ quickly drops to $8 \%$ suggesting the switching time is in between $5 / 8$ and $5 / 16 \mathrm{~s}$. This is an encouraging result as it shows that 1) PEDOT:PSS can serve as a proton source in an ECD and 2) it can yield comparable switching time to the liquid proton sources, which is typically in the sub-second level. ${ }^{16,17}$

Even though the switching speed by using protons as insertion ions is fast, the small $\Delta T$ of $15 \%$ is not sufficient for electrochromic applications. We attributed this small $\Delta T$ to the limited amount of removable protons in the PEDOT:PSS layer. To solve this problem, we design a tandem structure by adding an extra SPE layer containing $\mathrm{NaClO}_{4}$ and ferrocene on top of the PEDOT:PSS layer with an aim to use $\mathrm{Na}^{+}$ions from the SPE layer to pump more protons to the $\mathrm{WO}_{3}$ layer. Here, ferrocene was added into the electrolyte as a redox mediator in the ECD to compensate the charge lost due to $\mathrm{Na}^{+}$ ion extraction. ${ }^{34}$ This design is motivated by the fact that $\mathrm{NaClO}_{4}$-containing SPE is a widely used $\mathrm{Na}^{+}$source in $\mathrm{WO}_{3}$-based ECDs and meanwhile PSS is a well-known ionexchange material. ${ }^{35-37}$

Before fabricating a full device, we first examined the response time of the SPE/PEDOT:PSS junction to voltage pulses of different widths. We used a device structure of ITO/SPE/PEDOT:PSS/ITO. The lower panel of Fig. 1b shows the $\Delta T$ as a 
function of voltage pulse width. It can be seen that this device without the $\mathrm{WO}_{3}$ layer also exhibits electrochromic behavior. At pulse width of $40 \mathrm{~s}, \Delta T$ is $30 \%$. When the pulse width is reduced to $5 / 4 \mathrm{~s}, \Delta T$ still maintains at $28 \%$. When the pulse width is further reduced to $5 / 8 \mathrm{~s}, \Delta T$ drops to $18 \%$, suggesting that the SPE/PEDOT:PSS junction also has a switching time at sub-second level and is suitable for use in a tandem structure with the PEDOT:PSS/WO 3 junction.

Fig. 1c shows a schematic diagram for the tandem structure with five layers, i.e., ITO/SPE/PEDOT:PSS/WO $/$ /ITO. The expected working mechanism is that the protons are the main insertion ions to the $\mathrm{WO}_{3}$ layer and responsible for the coloration. The SPE layer only provides $\mathrm{Na}^{+}$ions to the PEDOT:PSS layer, but the $\mathrm{Na}^{+}$ions do not diffuse into $\mathrm{WO}_{3}$ in a significant amount. Instead, the $\mathrm{Na}^{+}$ions mainly play the role of pumping protons out of PEDOT:PSS through an ion-exchange process.

To justify this expected mechanism, we first carried out density functional theory calculations. We compared the cases with either a $\mathrm{Na}^{+}$ion or a proton from the PSS model injected into $\mathrm{WO}_{3}$, as shown in the Fig. 1c. We found that the insertion of $\mathrm{Na}^{+}$ into $\mathrm{WO}_{3}$ is $0.46 \mathrm{eV}$ higher in energy than a proton. The high coordination of $\mathrm{Na}$ atoms by $\mathrm{O}$ atoms from two side chains of PSS (see Fig. 1c and Fig. S1 in the SI) plays the key role of stabilizing a $\mathrm{Na}^{+}$ion in PSS over a proton.

We further verified this process by carrying out X-ray photoelectron spectroscopy (XPS) experiments on three different samples as shown in Fig. 1d, where both the $\mathrm{SPE} / \mathrm{WO}_{3}$ and SPE/PEDOT:PSS/WO ${ }_{3}$ samples were prepared after the coloration by ion insertion. Before the XPS experiments, all capping layers above $\mathrm{WO}_{3}$ were removed. Consistent with the calculation results, the contents of $\mathrm{Na}$ show obvious differences among these samples. Compared to SPE/ $\mathrm{WO}_{3}$, the significantly less $\mathrm{Na}$ content in the 
colored $\mathrm{WO}_{3}$ from the SPE/PEDOT:PSS/ $\mathrm{WO}_{3}$ sample indicates that the coloration of $\mathrm{WO}_{3}$ is caused by proton insertion.
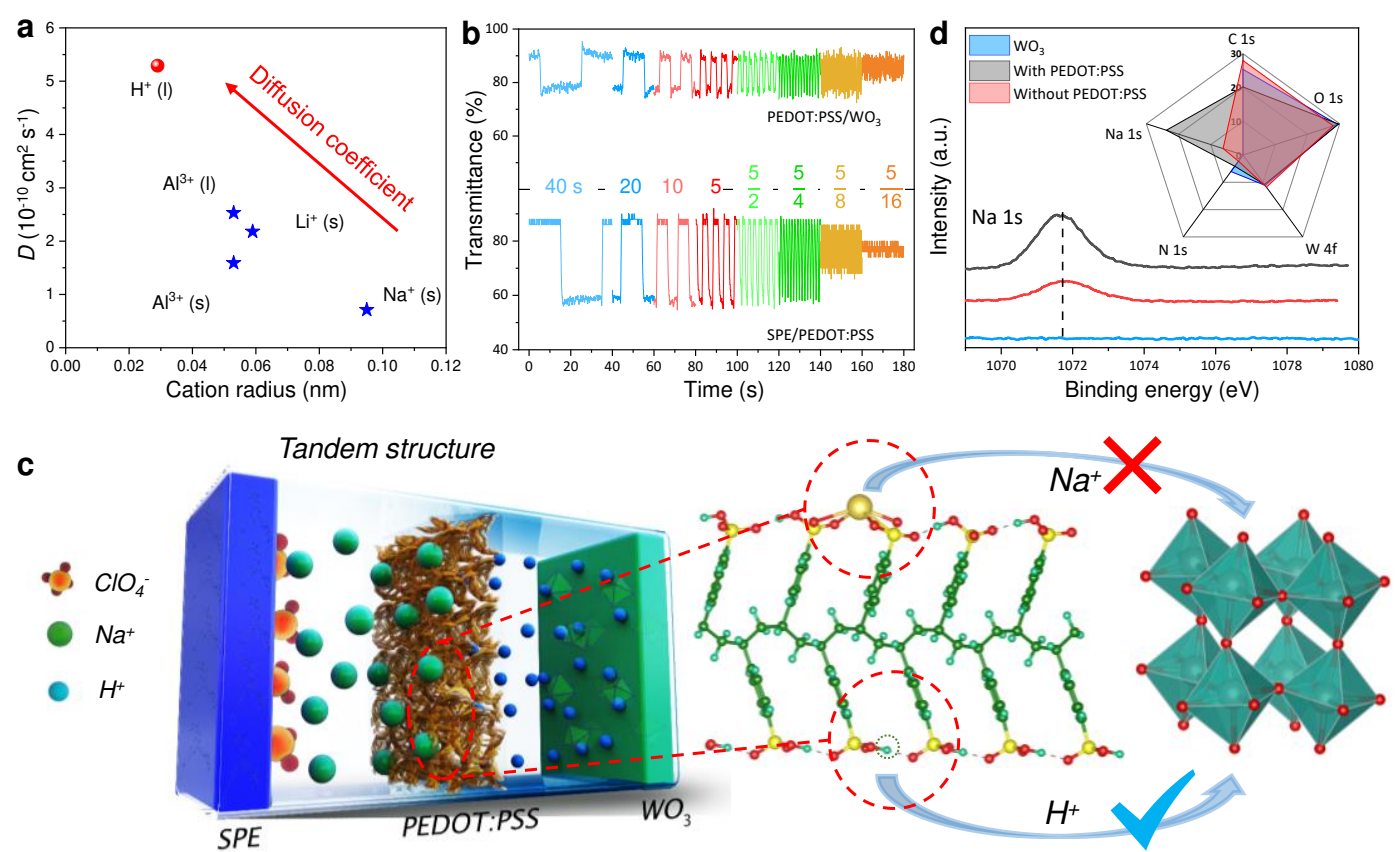

Fig. 1 | Design of the tandem-structure ECD. (a) Diffusion coefficient and radius for different inserting cations in $\mathrm{WO}_{3}$; (b) Transmittance changes at $670 \mathrm{~nm}$ with decreasing width of voltage pulses from 40 to 5/16 seconds, where the upper panel is from a PEDOT:PSS/ $\mathrm{WO}_{3}$ junction and the lower panel is from a SPE/PEDOT:PSS junction; (c) Schematic for the tandem structure ECD and the key step with a $\mathrm{Na}^{+}$ ion from the SPE layer pumping out a proton from PEDOT:PSS to $\mathrm{WO}_{3}$. (d) XPS spectra of $\mathrm{Na}$ 1s from three $\mathrm{WO}_{3}$ samples obtained from $\mathrm{WO}_{3}, \mathrm{SPE} / \mathrm{WO}_{3}$ and $\mathrm{SPE} / \mathrm{PEDOT}: \mathrm{PSS} / \mathrm{WO}_{3}$ devices. The inset shows the comparison of the contents of different elements from the three samples.

Characterization of the tandem-structure ECD. Fig. 2a and Fig. $2 b$ shows the schematic of the all-solid-state tandem structure device and the scanning electron microscope (SEM) cross-section image. More details about preparation and characterization of the SPE layer are given in Fig. S2 in the SI. Fig. 2c shows the photographs of the device under different applied voltages. Fig. $2 d$ shows the corresponding transmittance spectra. When the device is in the off state $(0 \mathrm{~V}$ applied voltage), the transmittance in the visible $(400-750 \mathrm{~nm})$ and NIR region $(750-1360 \mathrm{~nm})$ 
can exceed $90 \%$ and $85 \%$, respectively, in our best-performed devices. After applying a positive voltage of $1.2 \mathrm{~V}$, the transmittance is reduced to $\sim 30 \%$ and $\sim 40 \%$, respectively, in the visible and NIR region. When the voltage is further increased to 2.4 $\mathrm{V}$, the transmittance reaches $2.7 \%$ and $8.7 \%$ in the visible and NIR regions, respectively, which shows a much better optical modulation than the device without the PEDOT:PSS layer (above $20 \%$ in both visible and NIR region, see Fig. S3 in the SI). These results are consistent with the absorption spectra (Fig. S4 in the SI), where the absorption peaks are located at $620 \mathrm{~nm}$ and $860 \mathrm{~nm}$.

Fig. 2e shows the voltage dependence of the transmittance of the devices with and without the PEDOT:PSS layer. Clearly, the device with the PEDOT:PSS layer exhibits two stages in both the coloration and bleaching processes. Fig. $2 \mathrm{f}$ and Fig. $2 \mathrm{~g}$ compare the cyclic voltammetry $(\mathrm{CV})$ curves of the two devices, where two redox peaks were observed in the device with the PEDOT:PSS layer at all scan rates. In contrast, the device without the PEDOT:PSS layer only shows a single redox peak. We also carried out $\mathrm{x}^{*} \mathrm{y}^{*}$ color space (CIE 1931) measurements. As shown in Fig. S5 in the SI, the device with the PEDOT:PSS layer exhibits a shallow and a deep coloration state.

As determined above from DFT calculation and XPS measurement, the coloration of $\mathrm{WO}_{3}$ is mainly the result of proton insertion. We attribute the first stage of coloration to proton insertion. Due to the fact that the PEDOT:PSS layer is electrochromic by itself, we can attribute the second stage of coloration to $\mathrm{Na}^{+}$accumulation in the PEDOT: PSS layer, as further elaborated below. In the second stage, the voltage is as high as $2.4 \mathrm{~V}$. No pure proton sources can survive at this high voltage. The highest applied voltage on liquid proton source as reported in the literature is $1.5 \mathrm{~V}$ only. ${ }^{9}$ Fig. $2 \mathrm{~h}$ shows the measured voltage drops at individual layers, where the voltage drops on the SPE $\left(\mathrm{U}_{1}\right)$ 
and $\mathrm{WO}_{3}\left(\mathrm{U}_{2}\right)$ layers are measured directly, as shown in the inset of Fig. $2 \mathrm{~h}$, and the voltage drop on the PEDOT:PSS $\left(\mathrm{U}_{3}\right)$ layer is obtained by taking the difference between applied voltage and the sum of $U_{1}$ and $U_{2}$. It can be seen that $U_{3}$ is always below 0.35 V, which should prevent $\mathrm{H}_{2}$ gas from evolution. In Fig. $2 \mathrm{~h}$, it is also observed that $\mathrm{U}_{3}$ exhibits a peak at about $1.6 \mathrm{~V}$, which is consistent with the voltage onset for the second coloration stage in Fig. 2e. To confirm this behavior, we fabricated three such samples and similar peak position was observed (see Fig. S6a-d in SI). We attribute the second stage of coloration to $\mathrm{Na}^{+}$extraction from the SPE layer and injection to the PEDOT:PSS layer at the high voltage. The extra $\mathrm{Na}^{+}$ions result in the coloration of the PEDOT:PSS layer.

To understand the performance difference with and without the PEDOT:PSS layer, Fig. 2i compares the diffusion coefficients $(D)$ of the two devices determined from the data in Fig. 2f and Fig. $2 \mathrm{~g}$ using the Randles-Sevcik equation ${ }^{38}$ :

$$
i_{p}=2.69 \times 10^{5} A n^{3 / 2} D^{1 / 2} c v^{1 / 2}
$$

where $D$ is in the unit of $\mathrm{cm}^{2} \cdot \mathrm{s}^{-1}, i_{p}$ is the peak current (in $\mathrm{mA}$ ), $A$ is the working electrode area $\left(\mathrm{in} \mathrm{cm}^{2}\right), c$ is the concentration of active ion $\left(\mathrm{mol} \cdot \mathrm{cm}^{-3}\right), v$ is the scan rate $\left(\mathrm{mV} \cdot \mathrm{s}^{-1}\right), n$ is the number of electrons assumed to be 1 . In the case without the PEDOT:PSS layer, the diffusion current is contributed to $\mathrm{Na}^{+}$insertion from SPE to $\mathrm{WO}_{3}$. In the case with the PEDOT:PSS layer, the diffusion current is mainly contributed by proton insertion from PEDOT:PSS to $\mathrm{WO}_{3}$, which should be accompanied by $\mathrm{Na}^{+}$ insertion from SPE to PEDOT:PSS to fill the $\mathrm{H}$ vacancies, as shown in Fig. 1c. As expected, the kinetics of proton diffusion is indeed significantly faster than $\mathrm{Na}^{+}$ diffusion, which explains the enhanced performance by using the PEDOT:PSS layer. 


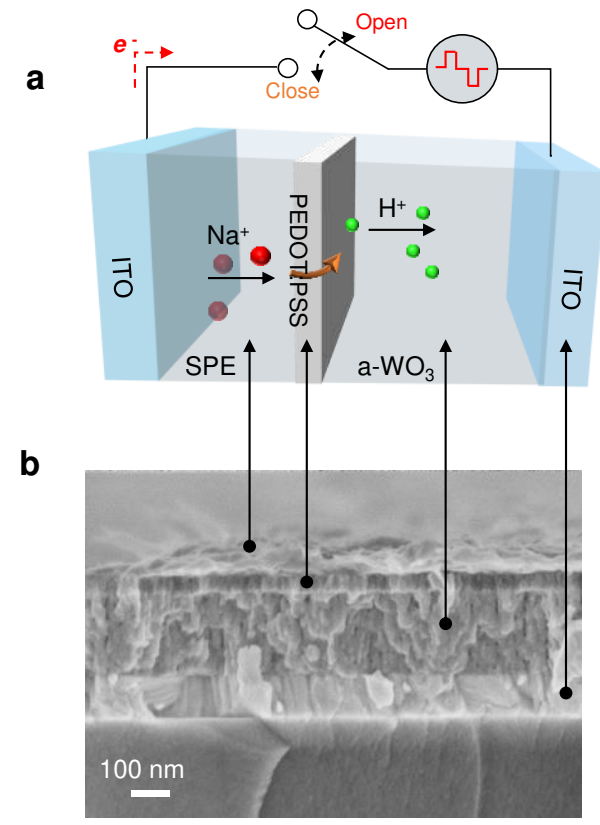

C

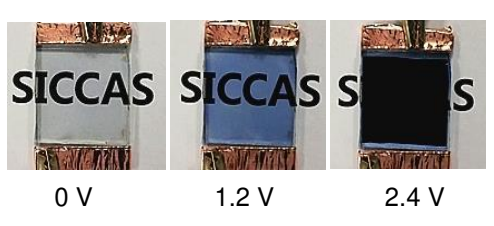

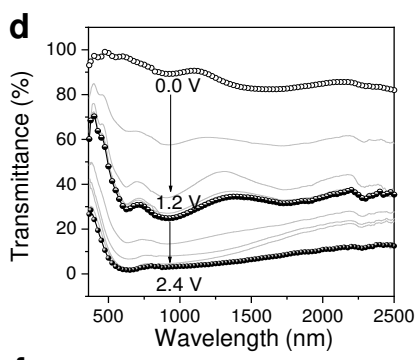
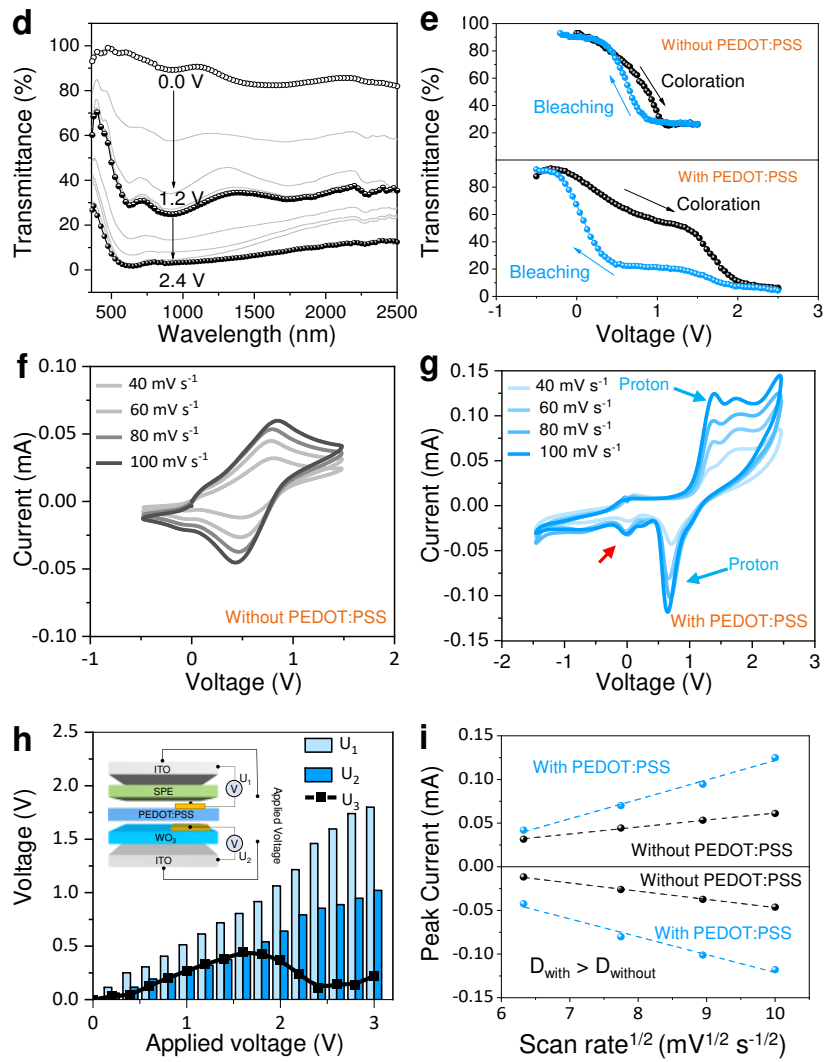

Fig. 2 Characterization of the tandem-structure ECD. (a) Schematic of the tandem structure ECD with five layers; (b) SEM image of the cross section of the device; (c) Photographs of the device at 0 , 1.2 and $2.4 \mathrm{~V}$ applied voltages; (d) Transmittance spectra of the device at three applied voltage; (e) Transmittance vs. voltage plots for the device without (upper) and with PEDOT:PSS (lower) at a scanning rate of $10 \mathrm{mV} / \mathrm{s}$ of the applied voltage; (f)-(g) $\mathrm{CV}$ curves of $\mathrm{SPE} / \mathrm{WO}_{3}$ and SPE/PEDOT:PSS/ $/ \mathrm{WO}_{3}$ devices at different scan rates; (h) Voltage drops at individual layers, where the voltage drops on the SPE $\left(\mathrm{U}_{1}\right)$ and $\mathrm{WO}_{3}\left(\mathrm{U}_{2}\right)$ layers are measured directly and the voltage drop on the PEDOT:PSS layer $\left(\mathrm{U}_{3}\right)$ is obtained by taking the difference between applied voltage and the sum of $\mathrm{U}_{1}$ and $U_{2}$. (i) Plots of the peak current densities vs. square root of scan rates using the results from (f) and (g). For the case with PEDOT:PSS, the peaks marked by blue arrows in (g) were used.

Performance of the tandem-structure ECD. We next examined the cycle life and switching time of the all-solid-state tandem structure ECD. Real-time transmittance spectra were taken at $-0.5 \mathrm{~V}$ and $2.5 \mathrm{~V}$ and the period for each cycle was set to 20 
seconds ensuring full coloration and bleaching. The result is shown in Fig. 3a. It can be seen that after 3000 cycles the device still retains over $90 \%$ of its initial optical modulation (i.e., less than $10 \%$ degradation). The inset of Fig. 3a shows the transmittance spectra of the 1000th, 2000th, and 3000th cycles confirming the consistency over the cycling. In contrast, the traditional ECD based on the simple SPE/WO $\mathrm{W}_{3}$ structure not only has a slow switching speed $(\sim 3.9 \mathrm{~s}$ for coloration and $\sim 9.8$ s for bleaching as shown in Fig. S7 in the SI), but also poor cycling stability suggesting that protons introduce less damage to the lattice than the larger $\mathrm{Na}^{+}$ions during the cycling.

Fig. $3 b$ shows the transmittance spectra for a typical cycle from which we determine the switching times for the coloration and bleaching processes. We define the coloration time $\left(t_{\mathrm{c}}\right)$ and bleaching time $\left(t_{\mathrm{b}}\right)$ as the time taken for the relative change in transmittance reaching $90 \%$. Our results show that $t_{\mathrm{c}}$ is about $0.7 \mathrm{~s}$ at applied voltage of $2.5 \mathrm{~V}$ while $t_{\mathrm{b}}$ is $7.1 \mathrm{~s}$ at $-0.5 \mathrm{~V}$. However, an inspection of the spectrum shows that the bleaching process exhibits two stages. The first stage with the relative change in transmittance reaching $65 \%$ only takes $0.9 \mathrm{~s}$. It is the second stage that is significantly slower. This slow second stage is attributed to the strong affiliation of $\mathrm{Na}^{+}$in the PEDOT:PSS layer. From the CV measurement (the red-colored arrow in Fig. $2 \mathrm{~g}$ ), it can be seen that the peak current is nearly unchanged for high scan rates indicating that the second stage involves a slow ion diffusion process. From Fig. $2 \mathrm{~g}$, it can also be seen that the coloration also involves two peaks. But these two peaks show similar scan-rate dependence, i.e., having similar ion diffusion rates, which explains that the coloration in Fig. 3b does not exhibit two stages. As a comparison, the result for the traditional SPE/ $\mathrm{WO}_{3}$ structure without the PEDOT:PSS layer is also shown in Fig. $3 \mathrm{~b}$. The significant improvement by introducing the PEDOT:PSS layer can be clearly seen. 
It is worth noting that the PEDOT:PSS layer has an optimized thickness. If the layer is too thick, the intrinsic light absorption will reduce the transmittance in the off state as shown in Fig. S8a-f. If the layer is too thin, it cannot provide sufficient protons to the $\mathrm{WO}_{3}$ layer. We fabricated a number of devices with varying thickness of the PEDOT:PSS layer. The thickness was controlled by the times of spin coating. The thickness of the $\mathrm{WO}_{3}$ layer was fixed at about $300 \mathrm{~nm}$, while the thickness of the SPE layer is usually much thicker (see Fig. 2b) and therefore does not affect the device performance significantly. As shown in Fig. 3c, the thicker the PEDOT:PSS layer is, the faster the coloration speed is until reaching the intrinsic limit of proton diffusion, which is estimated from our results to be $0.7-0.9 \mathrm{~s}$ (specific process can be seen in Fig. S9). In the best-performed device, the PEDOT:PSS layer is of the order of $50 \mathrm{~nm}$ as shown in Fig. 2b, which matches with the 300-nm $\mathrm{WO}_{3}$ layer.

Another important performance indicator for an ECD is the coloration efficiency $(\mathrm{CE})$, defined as the optical density changes $(\Delta \mathrm{OD})$ per unit of charge injection $(\Delta \mathrm{Q})$. A high CE value indicates a large optical modulation with a small energy input. As determined from Fig. 3d, the CE for the tandem structure with PEDOT:PSS is $109 \mathrm{~cm}^{2}$ $\mathrm{C}^{-1}$ at $670 \mathrm{~nm}$, while the $\mathrm{CE}$ for the device without PEDOT:PSS is $93.5 \mathrm{~cm}^{2} \mathrm{C}^{-1}$. As a comparison, a typical $\mathrm{LiTaO}_{3}$-based ECD shows a CE of $73.5 \mathrm{~cm}^{2} \mathrm{C}^{-1}$ (see Fig. $\mathrm{S} 10$ in the SI).

Fig. 3e compares the tandem structure (with the PEDOT:PSS layer) and the traditional structure (without the PEDOT:PSS layer) on their responses to voltage change with different pulse widths. The transmittance was measured at $670 \mathrm{~nm}$. The initial $\Delta T$ with pulse width of $20 \mathrm{~s}$ is $\sim 87 \%$ and $\sim 70 \%$ for the tandem and traditional structure, respectively. As the pulse width is reduced to $10,5,5 / 2,5 / 4,5 / 8$, and $5 / 16 \mathrm{~s}$, 
the $\Delta T$ of the tandem structure decreases to $86 \%, 81 \%, 71 \%, 65 \%, 58 \%$, and $48 \%$, respectively, and the $\Delta T$ of the traditional structure decreases to $65 \%, 60 \%, 45 \%, 35 \%$, $25 \%$, and $15 \%$, respectively (Fig. S11 in the SI). This result further demonstrates the superiority of the tandem structure over the traditional structure from the aspect of optical modulation. The $17 \%$ increase in the $\mathrm{CE}$ can be attributed to the second coloration stage. It also suggests that the main contributor to coloration in the tandem structure is the $\mathrm{WO}_{3}$ layer, while the PEDOT:PSS mainly serves as an intermediary layer for the relay of protons and $\mathrm{Na}^{+}$ions. This is consistent with the results in Fig. 1 that without the synergy between the three layers in the tandem structure, the optical modulation is rather low.

A high-performance ECD is typically characterized by the switching time and the optical modulation (i.e., the maximum $\Delta T$ between on/off states). We summarize the optical modulation and switching time for a number of representative $\mathrm{WO}_{3}$-based ECDs in Fig. 3f. Proton-based devices are located at the top-left corner with fast switching speed and high optical modulation. Recent advances in ECDs based on $\mathrm{Al}^{3+}$ ions typically yield high optical modulation, but the switching speed is slow due to the diffusion of trivalent ions. ECDs based on $\mathrm{Li}^{+}$and $\mathrm{Na}^{+}$ions are usually not comparable with the proton-based devices. 

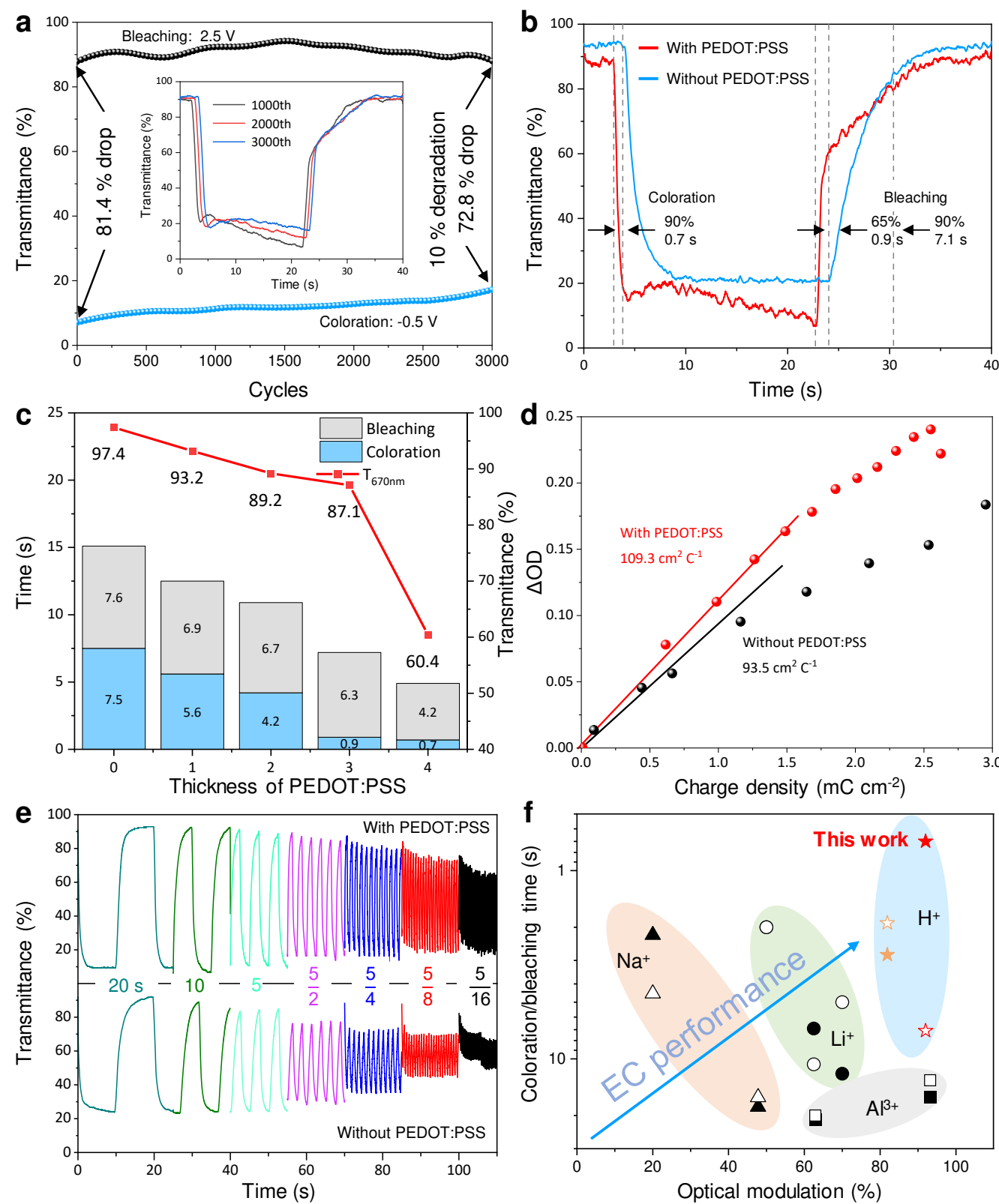

Fig. 3 Performance of the tandem-structure ECD. (a) Real-time transmittance spectra of ECD at 670 $\mathrm{nm}$ under -0.5 and $2.5 \mathrm{~V}$ voltage, respectively. The inset is the transmittance spectra of the $1000^{\text {th }}, 2000^{\text {th }}$ and $3000^{\text {th }}$ cycles; (b) Measurement of switching times from the transmittance spectra; (c) Relationship of the switching times and transmittance (at $670 \mathrm{~nm}$ ) with the thicknesses of the PEDOT:PSS layer. Here, the thickness was controlled by the times of spin coating. (d) Changes in optical density (at $670 \mathrm{~nm}$ ) with respect to injected charge density; (e) Response of transmittance to decreasing voltage pulse width from 20 to 5/16 seconds; (f) Switching time and optical modulation of $\mathrm{WO}_{3}$-based ECDs with various insertion ions. 
Scale-up of the tandem-structure ECD. A major advantage of all-solid-state ECDs is the convenience of fabrication and encapsulation for large-scale applications. The traditional inorganic electrolytes like $\mathrm{LiTaO}_{3}$ also suffer from low deposition rate which results in high cost of fabrication and low uniformity of the devices. Here, the polymerbased electrolytes in our tandem structure design can be easily prepared and scaled up. To test the scalability, we fabricated $10 \times 10 \mathrm{~cm}^{2}$ windows based on the tandem structure. Photographs in Fig. 4a show the uniformity of the coloration at different applied voltages as inspected by naked eyes. The transmittance changes at $670 \mathrm{~nm}$ recorded at the center and the edge of the window are shown in Fig. 4b. Full spectra are shown in Fig. S12 in the SI. At both regions the transmittance decreases from about $90 \%$ to about $20 \%$ in $2.1 \mathrm{~s}$ and $6.3 \mathrm{~s}$. Fig. $4 \mathrm{~b}$ also compares the large-area devices based on both the tandem structure and the traditional structure without the PEDOT:PSS layer. The solar irradiance converted from the transmittance spectra is shown in Fig. 4c and Fig. 4d, which demonstrate high optical modulation for the full solar spectrum. Besides, we also fabricated a $30 \times 40 \mathrm{~cm}^{2}$ window. As shown in Fig. 4e, uniform coloration can also be achieved (more detailed results are given Fig. S13 in the SI).

The tandem structure discussed above could be easily adapted to make flexible ECDs. By replacing the brittle ITO layers with Ag nanowires, the whole device can be fabricated on a polyethylene terephthalate (PET) film. The Ag nanowires (NWs) were transferred onto the PET film by a blade coating method. The $\mathrm{WO}_{3}$, PEDOT:PSS and SPE layers between the electrodes can be similarly coated (see Methods for detailed processes). The flexible ECD of $10 \times 10 \mathrm{~cm}^{2}$ under the on and off conditions are shown in Fig. 4f. The device works well when being bended suggesting great potential for flexible applications of the all-solid-state tandem structure ECD. 


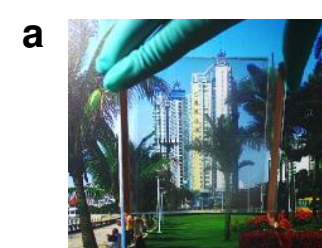

b 100 $0 \mathrm{~V}$
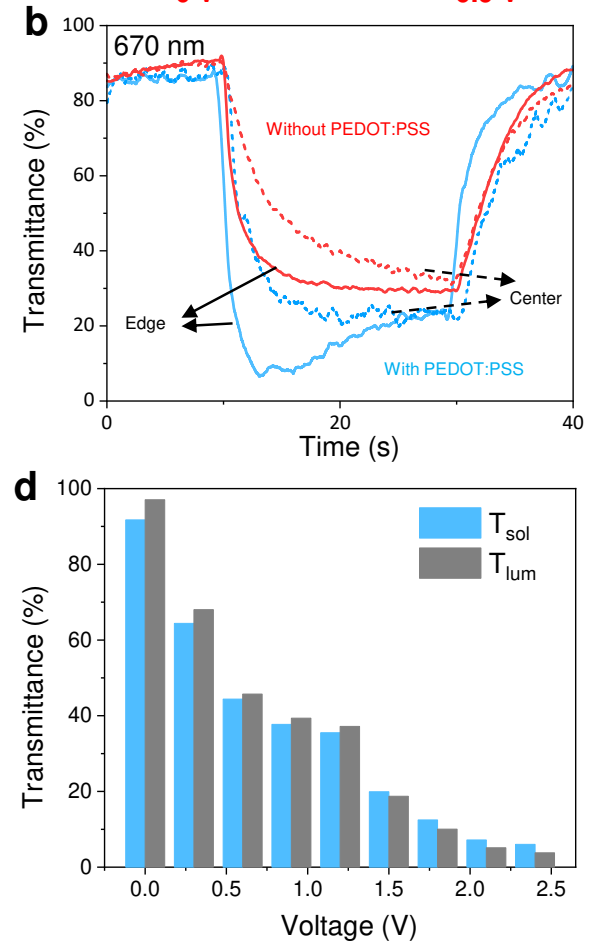

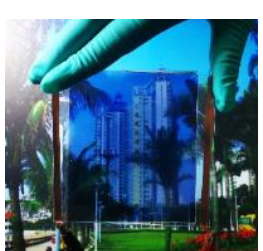

c

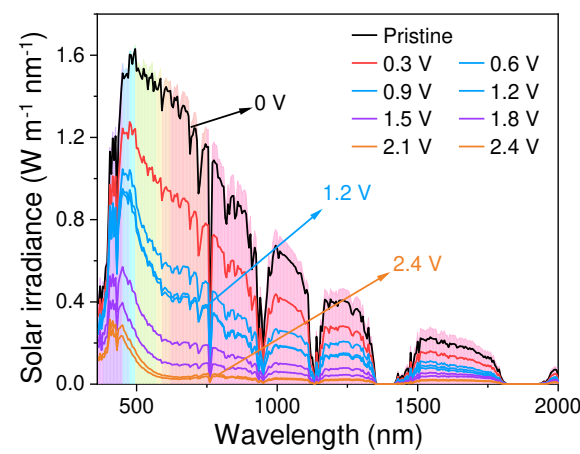

e

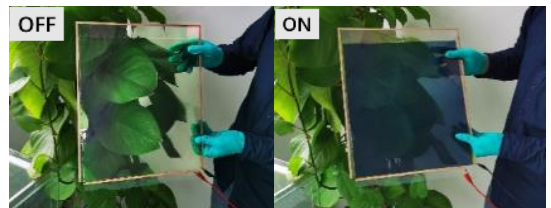

f

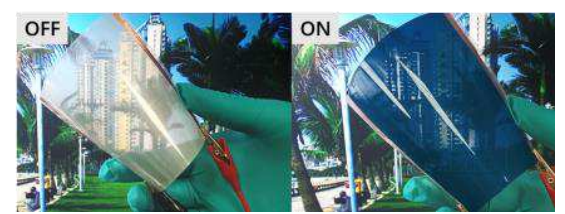

Fig. 4 | Scale-up of the tandem-structure ECD. (a) Photographs of a $10 \times 10 \mathrm{~cm}^{2}$ window under $0 \mathrm{~V}$, $0.5 \mathrm{~V}, 1.5 \mathrm{~V}$ and $2.0 \mathrm{~V}$; (b) Transmittance at $670 \mathrm{~nm}$ at the edge (blue) and the center (red) of the window during switching at $2 \mathrm{~V}$ for $20 \mathrm{~s}$ followed by $-1 \mathrm{~V}$. For comparison, the results from a device of the same size but without the PEDOT:PSS layer are also shown; (c) Solar irradiance spectra converted from the measured transmittance spectra of the device; (d) Transmittance vs. voltage results for the all-solid-state EC device (blue bar for solar modulation ability and black bar for luminous transmittance). (e) Photographs of a $30 \times 40 \mathrm{~cm}^{2}$ ECD; (f) Photographs of a flexible ECD.

\section{Conclusion}

We introduced PEDOT:PSS as a solid-state proton source for electrochromism in $\mathrm{WO}_{3}$, which enables an all-solid-state design of proton-based ECDs. To effectively extract protons from PEDOT:PSS, which is critical for enhancing the optical modulation of the ECDs, we further designed a tandem structure of SPE/PEDOT:PSS/ $\mathrm{WO}_{3}$, which allows 
a relay process of $\mathrm{Na}^{+}$ions and protons in the PEDOT:PSS layer, i.e., using $\mathrm{Na}^{+}$ions from the SPE layer to pump more protons from PEDOT:PSS to $\mathrm{WO}_{3}$. Overall, the ECD based on the all-solid-state tandem structure achieved ultrafast response (coloration to $90 \%$ in $0.7 \mathrm{~s}$ and bleaching to $65 \%$ in $0.9 \mathrm{~s}$ and $90 \%$ in $7.1 \mathrm{~s}$ ), high optical modulation (about $90 \%$ ) and excellent durability ( $<10 \%$ degradation after 3000 cycles). We also demonstrated scale-up capability of the all-solid-state tandem structure by fabricating large-area ECDs up to $30 \times 40 \mathrm{~cm}^{2}$ with appealing performance. A two-stage electrochromism was observed in the tandem structure, which were dominated by proton insertion to $\mathrm{WO}_{3}$ and $\mathrm{Na}^{+}$insertion to PEDOT:PSS, respectively. This two-stage ion insertion not only helps enhance the optical modulation of the ECD, but may also find applications in, e.g., information displays and triple-state optical devices. 


\section{Experimental}

\section{Materials}

PEDOT: PSS, $\mathrm{NaClO}_{4}, \mathrm{PMA}$ (Propylene glycol methyl ether acetate), ferrocene and PC (propylene carbonate) were purchased from Alfa Aesar.

TTA21(252.31) was purchased from Tetra Co., Ltd.

1,2-Dimethyl-3-Propylimidazolium Iodide was purchased from Adamas.

Transparent indium tin oxide (ITO) glass electrode and flexible Ag NW-PET were purchased from CSG Holding Co., Ltd.

\section{Material and device characterization}

UV-vis transmittance/absorption spectra were measured using a Hitachi U-4100 spectrophotometer. FT-IR spectra were measured using a Thermo Fisher Scientific Nicolet iS10 spectrophotometer. Cyclic voltammograms and EIS were measured using a Zahner IME6 electrochemical work station. Wetting contact angles were measured using a JY-82A contact angle meter. The EC devices were analyzed by scanning electron microscopy (SEM) using a Hitachi SU8220 equipped with an energy dispersive X-ray spectrometer (EDS) from Oxford X-Max ${ }^{\mathrm{N}}$.

\section{Preparation of $\mathrm{WO}_{3}$}

The amorphous $\mathrm{WO}_{3}$ layer was deposited by re-active DC magnetron sputtering for 30 min at a power of $70 \mathrm{~W}$, an $\mathrm{Ar} / \mathrm{O}_{2}$ ratio of $94: 6$ and a pressure of $2 \mathrm{~Pa}$.

\section{Preparation of solid polymer electrolyte (SPE)}

$\mathrm{NaClO}_{4} / \mathrm{PC}(1 \mathrm{~mol} / \mathrm{L})$, ferrocene and TTA21 in a ratio of 1:0.1:1 $\mathrm{g}$ were mixed with 2 $\mathrm{mL}$ of PMA and then stirred for $30 \mathrm{~min}$. After that, $0.01 \mathrm{~g}$ iodonium salt (1,2-Dimethyl3-Propylimidazolium Iodide) was added into the mixtures and stirred for another $5 \mathrm{~min}$. The pristine electrolyte precursor was a gel-like mixture $(2 \mathrm{~mL})$, which was spin-coated (1000 rpm, $20 \mathrm{~s}$ ) on the ITO/quartz electrode and then capped by another electrode. 
After curing for 30 seconds by a 300-W UV lamp, the electrolyte is solidified and meanwhile binds the two ITO layers. Here, $\mathrm{NaClO}_{4}$ dissolved in $\mathrm{PC}$ was used as a $\mathrm{Na}^{+}$ cation source. Ferrocene was used as a reducing (charge-compensating) agent in the electrochromic process. TTA21 and iodonium salt (1,2-Dimethyl-3-Propylimidazolium Iodide, Adamas) were used as UV-curing monomer and initiator.

\section{Preparation of PEDOT: PSS}

PEDOT: PSS dispersion was spun onto the bottom $\mathrm{ITO} / \mathrm{WO}_{3}$ electrode at $1000 \mathrm{rpm}$ for $60 \mathrm{~s}$, followed by $3000 \mathrm{rpm}$ for $20 \mathrm{~s}$. Then the substrates were heated to $80{ }^{\circ} \mathrm{C}$ with a rate of $10{ }^{\circ} \mathrm{C} / \mathrm{min}$ followed by annealing under vacuum condition.

\section{First-principles calculation}

The first-principles calculation was implemented in VASP program. ${ }^{39}$ Projector augmented wave (PAW) potentials were used to describe the core-valence interaction. ${ }^{40}$ Plane waves with kinetic energy up to $408 \mathrm{eV}$ were used as the basis set. PerdewBurke-Ernzerhof (PBE) functional ${ }^{41}$ was used in the calculation. All the structures were relaxed until the force on each atom was less than $0.03 \mathrm{eV} / \AA$ and then the energy was calculated. Structural models are described in Fig. S1 in SI. 


\section{ASSOCIATED CONTENT}

\section{AUTHOR INFORMATION}

X.C. conceived the project. Z.S., Y.Y.S., X.C. and A.H. designed the experiments and analyzed the data. Z.S., L.J., L.M., and A.H. performed the experiments and some characterizations. Z.S. performed optical simulations under the supervision of P.J., J. B. and H.L. Y.Y.S., C.M., and Z.S. conceived the device working mechanism, conducted the computational studies and data analysis. Z.S. and Y.Y.S. wrote the paper. All authors discussed the results and commented on the manuscript.

\#These authors contributed equally to this work.

*Corresponding Authors: cxun@mail.sic.ac.cn (X.C), hongjieluo@ shu.edu.cn (H.L.), yysun@mail.sic.ac.cn (Y.Y.S.)

\section{NOTES}

The authors declare no competing financial interest.

\section{ACKNOWLEDGMENTS}

This study was financially supported by the National Natural Science Foundation of China (No. 51572284, No. 51972328, No. 62005301 and No. 11774365), the Youth Innovation Promotion Association, Chinese Academy of Sciences (No. 2018288), Shanghai Sailing Program (No. 19YF1454300, No. 20YF1455400), the Shanghai Pujiang Program (No. 18PJD051) and the Key Research and Development Plan of Anhui Province (1804a09020061). 


\section{Reference:}

1. M. T. Strand et al., Factors That Determine the Length Scale for Uniform Tinting In Dynamic Windows Based on Reversible Metal Electrodeposition. ACS Energy Letters 3, 2823-2828 (2018).

2. P. M. Monk, R. J. Mortimer, D. R. Rosseinsky, Electrochromism and Electrochromic Devices. (Cambridge University Press Cambridge, 2007)

3. C. G. Granqvist, Electrochromic Tungsten Oxide Films: Review of Progress 19931998. Solar Energy Materials and Solar Cells 60, 201-262 (2000).

4. C.-G. Granqvist, Handbook of Inorganic Electrochromic Materials. (1995).

5. S.-H. Lee et al., Crystalline $\mathrm{WO}_{3}$ Nanoparticles for Highly Improved Electrochromic Applications. Advanced Materials 18, 763-766 (2006).

6. S. Sallard, T. Brezesinski, B. M. Smarsly, Electrochromic Stability of $\mathrm{WO}_{3}$ Thin Films with Nanometer-Scale Periodicity and Varying Degrees of Crystallinity. Journal of Physical Chemistry C 111, 7200-7206 (2007).

7. P. Cossari et al., Simplified All-Solid-State $\mathrm{WO}_{3}$ Based Electrochromic Devices on Single Substrate: Toward Large Area, Low Voltage, High Contrast, and Fast Switching Dynamics. Advanced Materials Interfaces 7, 1901663 (2020).

8. J.-G. Zhang et al., Chromic Mechanism In Amorphous $\mathrm{WO}_{3}$ Films. J. Electrochem. Soc. 144, 2022-2026 (1997).

9. K. J. Patel, C. J. Panchal, M. S. Desai, P. K. Mehta, An Investigation of the Insertion of the Cations $\mathrm{H}^{+}, \mathrm{Na}^{+}, \mathrm{K}^{+}$on the Electrochromic Properties of the Thermally Evaporated $\mathrm{WO}_{3}$ Thin Films Grown at Different Substrate Temperatures. Materials Chemistry and Physics 124, 884-890 (2010).

10. S.-H. Lee et al., Electrochromic Coloration Efficiency of a- $\mathrm{WO}_{3-\gamma}$ Thin Films as $\mathrm{A}$ Function of Oxygen Deficiency. Applied Physics Letters 75, 1541-1543 (1999).

11. S. Y. Lee et al., Electrochromic Mechanism in A-WO\{Sub 3 \{minus\}y\} Thin Films. Applied Physics Letters 74, (1999).

12. J. Guo et al., Prominent Electrochromism Achieved Using Aluminum Ion Insertion/Extraction In Amorphous $\mathrm{WO}_{3}$ Films. Journal of Physical Chemistry C122, 19037-19043 (2018).

13. S. Zhang et al., $\mathrm{Al}^{3+}$ Intercalation/De-Intercalation-Enabled Dual-Band Electrochromic Smart Windows with A High Optical Modulation, Quick Response and Long Cycle Life. Energy \& Environmental Science 11, 2884-2892 (2018).

14. Z. Tong et al., Recent Advances In Multifunctional Electrochromic Energy Storage Devices and Photoelectrochromic Devices. Science China Chemistry 60, 13-37 (2017).

15. D. Dini, F. Decker, E. Masetti, A Comparison of the Electrochromic Properties of WO3 Films Intercalated with $\mathrm{H}^{+}$, $\mathrm{Li}^{+}$and $\mathrm{Na}^{+}$. Journal of Applied Electrochemistry 26, 647-653 (1996).

16. Y. Tian et al., Unconventional Aluminum Ion Intercalation/Deintercalation for Fast Switching and Highly Stable Electrochromism. Advanced Functional Materials 25, 5833-5839 (2015).

17. C. Yan et al., Stretchable and Wearable Electrochromic Devices. ACS Nano 8, 316322 (2014). 
18. J. Z. Ou et al., the Anodized Crystalline $\mathrm{WO}_{3}$ Nanoporous Network with Enhanced Electrochromic Properties. Nanoscale 4, 5980-5988 (2012).

19. G. Cai et al., Ultra-Large Optical Modulation of Electrochromic Porous $\mathrm{WO}_{3}$ Film and the Local Monitoring of Redox Activity. Chemical Science 7, 1373-1382 (2016).

20. T. C. Arnoldussen, A Model for Electrochromic Tungstic Oxide Microstructure and Degradation. J. Electrochem. Soc. 128, 117-123 (1981).

21. Y. Zhu et al., High Performance All-Solid-State Electrochromic Device Based on Lixnioy Layer with Gradient Li Distribution. Electrochimica Acta 317, 10-16 (2019).

22. S. J. Yoo, J. W. Lim, Y.-E. Sung, Improved Electrochromic Devices with An Inorganic Solid Electrolyte Protective Layer. Solar Energy Materials and Solar Cells 90, 477 484 (2006).

23. S. J. Yoo et al., Fast Switchable Electrochromic Properties of Tungsten Oxide Nanowire Bundles. Applied Physics Letters 90, 173126 (2007).

24. H. M. Huang et al., Quasi-Hodgkin-Huxley Neurons with Leaky Integrate-AndFire Functions Physically Realized with Memristive Devices. Advanced Materials 31, 1803849 (2019).

25. Y. van de Burgt et al., A Non-Volatile Organic Electrochemical Device As A LowVoltage Artificial Synapse for Neuromorphic Computing. Nature Materials 16, 414-418 (2017).

26. G. Cai et al., Direct Inkjet-Patterning of Energy Efficient Flexible Electrochromics. Nano Energy 49, 147-154 (2018).

27. Y. Yue et al., High-Performance Complementary Electrochromic Device Based on $\mathrm{WO}_{3} \cdot 0.33 \mathrm{H}_{2} \mathrm{O} / \mathrm{PEDOT}$ and Prussian Blue Electrodes. Journal of Physics and Chemistry of Solids 110, 284-289 (2017).

28. C. Dulgerbaki, N. N. Maslakci, A. I. Komur, A. U. Oksuz, PEDOT/WO $\mathrm{W}_{3}$ Hybrid Nanofiber Architectures for High Performance Electrochromic Devices. Electroanalysis 28, 1873-1879 (2016).

29. D. Delongchamp, P. T. Hammond, Layer-By-Layer Assembly of PEDOT/Polyaniline Electrochromic Devices. Advanced Materials 13, 1455-1459 (2001).

30. R. Singh et al., ITO-Free Solution-Processed Flexible Electrochromic Devices Based on PEDOT:PSS as Transparent Conducting Electrode. ACS Applied Materials \& Interfaces 9, 19427-19435 (2017).

31. C. L. Gaupp et al., Composite Coloration Efficiency Measurements of Electrochromic Polymers Based on 3,4-Alkylenedioxythiophenes. Chemistry of Materials 14, 3964-3970 (2002).

32. E. Poverenov, M. Li, A. Bitler, M. Bendikov, Major Effect of Electropolymerization Solvent on Morphology and Electrochromic Properties of PEDOT Films. Chemistry of Materials 22, 4019-4025 (2010).

33. B. Deng et al., Roll-to-Roll Encapsulation of Metal Nanowires between Graphene and Plastic Substrate for High-Performance Flexible Transparent Electrodes. nano letters 15, 4206-4213 (2015).

34. H.-C. Lu et al., An Electrochromic Device Based on Prussian Blue, Self-Immobilized Vinyl Benzyl Viologen, and Ferrocene. Solar Energy Materials and Solar Cells 147, 75-84 (2016).

35. S.-L. Chen et al., Ion Exchange Resin/Polystyrene Sulfonate Composite 
Membranes for PEM Fuel Cells. Journal of Membrane Science 243, 327-333 (2004).

36. K.-M. Mangold et al., Ion Exchange Properties and Selectivity of PSS In An Electrochemically Switchable Ppy Matrix. Journal of Applied Electrochemistry 35, 1293-1301 (2005).

37. S.-L. Chen et al., Ion Exchange Resin/Polystyrene Sulfonate Composite Membranes for PEM Fuel Cells. Journal of Membrane Science 243, 327-333 (2004).

38. D. A. Skoog, F. J. Holler, T. A. Nieman, Principles of Instrumental Analysis, 6th edn. (Cengage Learning, MA, 2006)

39. G. J. Kresse, Furthmller, Efficiency of Ab-Initio Total Energy Calculations for Metals and Semiconductors using a Plane-Wave Basis Set. Computational Materials Science 6, 15-50 (1996)

40. G. J. Kresse, D. Joubert, From Ultrasoft Pseudopotentials to the Projector Augmented-Wave Method. Physical Review B, 59, 1758 (1999).

41. J. P. Perdew, K. Burke, M. Ernzerhof, Generalized gradient approximation made simple. Physical Review Letters, 77, 3865 (1996). 


\section{Figures}
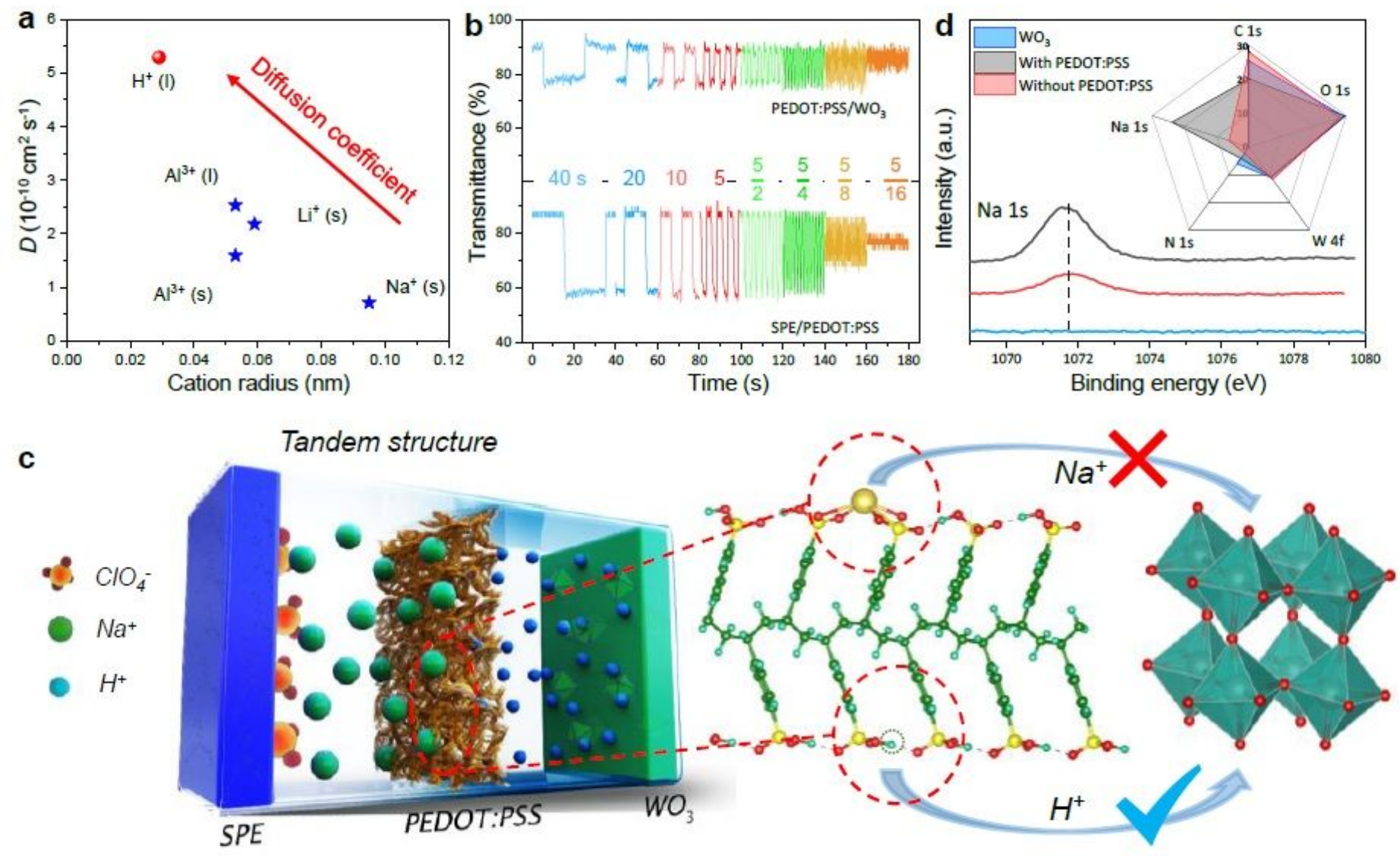

\section{Figure 1}

Design of the tandem-structure ECD. (a) Diffusion coefficient and radius for different inserting cations in WO3; (b) Transmittance changes at $670 \mathrm{~nm}$ with decreasing width of voltage pulses from 40 to 5/16 seconds, where the upper panel is from a PEDOT:PSS/WO3 junction and the lower panel is from a SPE/PEDOT:PSS junction; (c) Schematic for the tandem structure ECD and the key step with a Na+ ion from the SPE layer pumping out a proton from PEDOT:PSS to WO3. (d) XPS spectra of Na $1 \mathrm{~s}$ from three WO3 samples obtained from WO3, SPE/WO3 and SPE/PEDOT:PSS/WO3 devices. The inset shows the comparison of the contents of different elements from the three samples. 

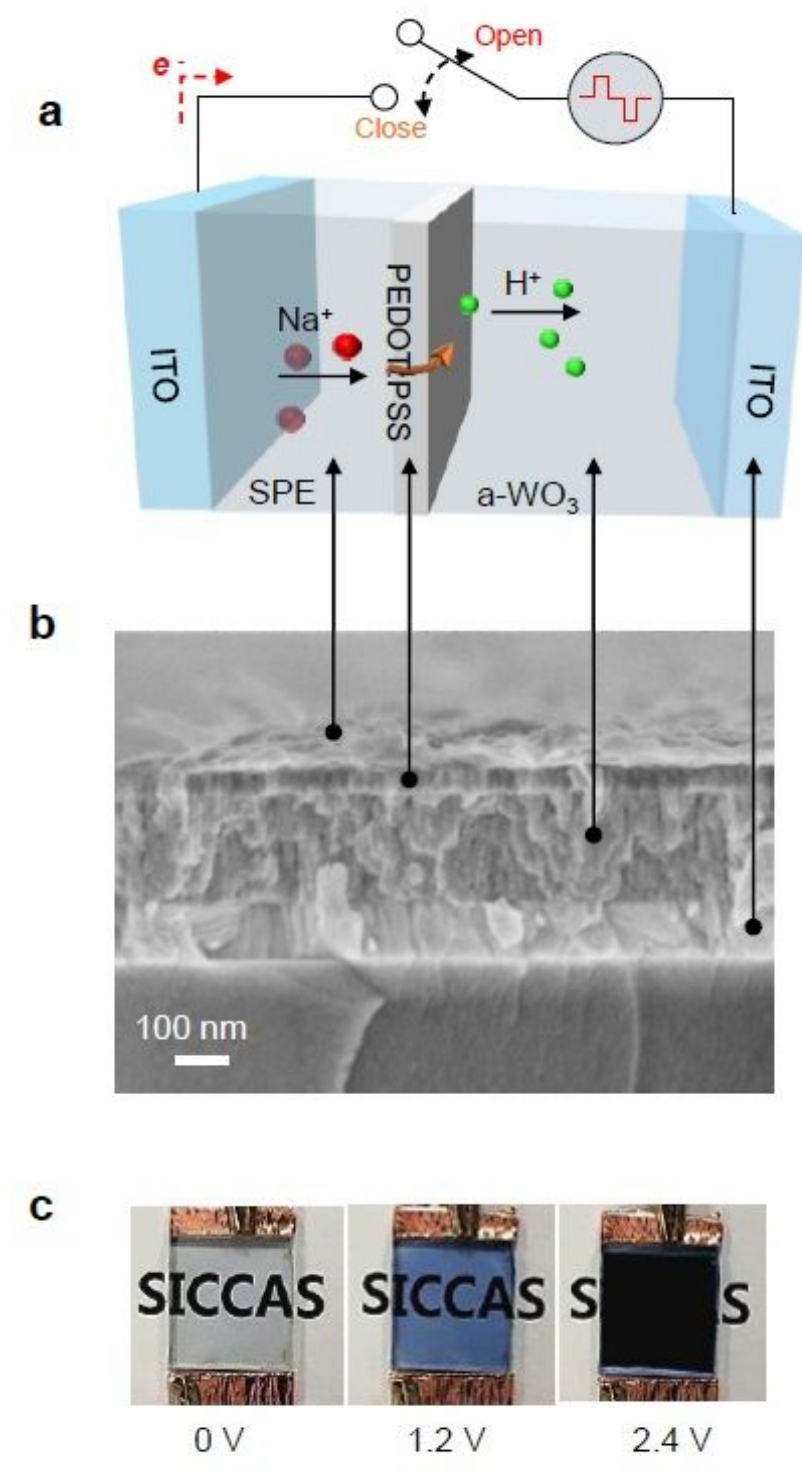
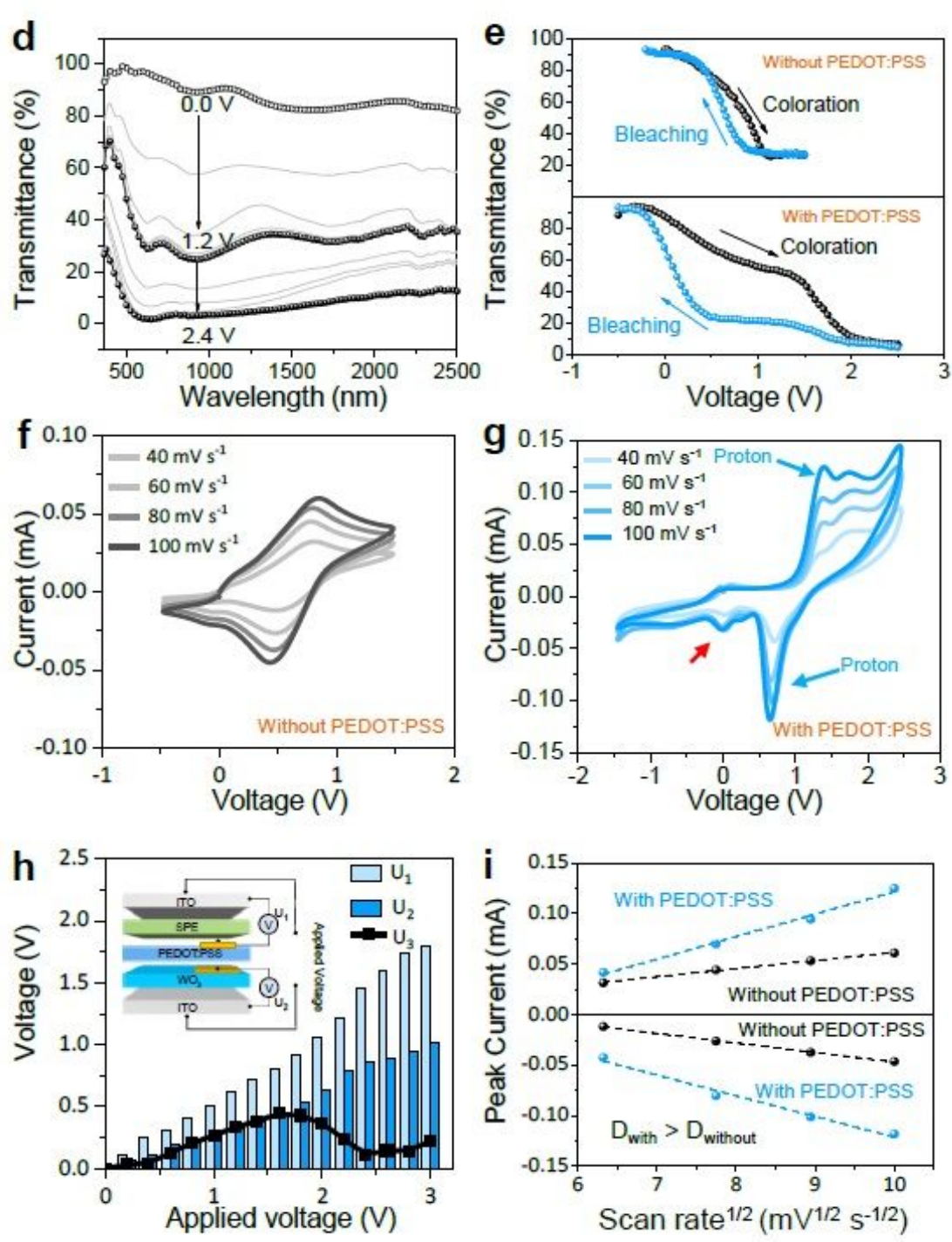

\section{Figure 2}

Characterization of the tandem-structure ECD. (a) Schematic of the tandem structure ECD with five layers; (b) SEM image of the cross section of the device; (c) Photographs of the device at 0,1.2 and $2.4 \mathrm{~V}$ applied voltages; (d) Transmittance spectra of the device at three applied voltage; (e) Transmittance vs. voltage plots for the device without (upper) and with PEDOT:PSS (lower) at a scanning rate of $10 \mathrm{mV} / \mathrm{s}$ of the applied voltage; (f)-(g) CV curves of SPE/WO3 and SPE/PEDOT:PSS/WO3 devices at different scan rates; (h) Voltage drops at individual layers, where the voltage drops on the SPE (U1) and WO3 (U2) layers are measured directly and the voltage drop on the PEDOT:PSS layer (U3) is obtained by taking the difference between applied voltage and the sum of U1 and U2. (i) Plots of the peak current densities vs. square root of scan rates using the results from $(f)$ and $(g)$. For the case with PEDOT:PSS, the peaks marked by blue arrows in (g) were used. 

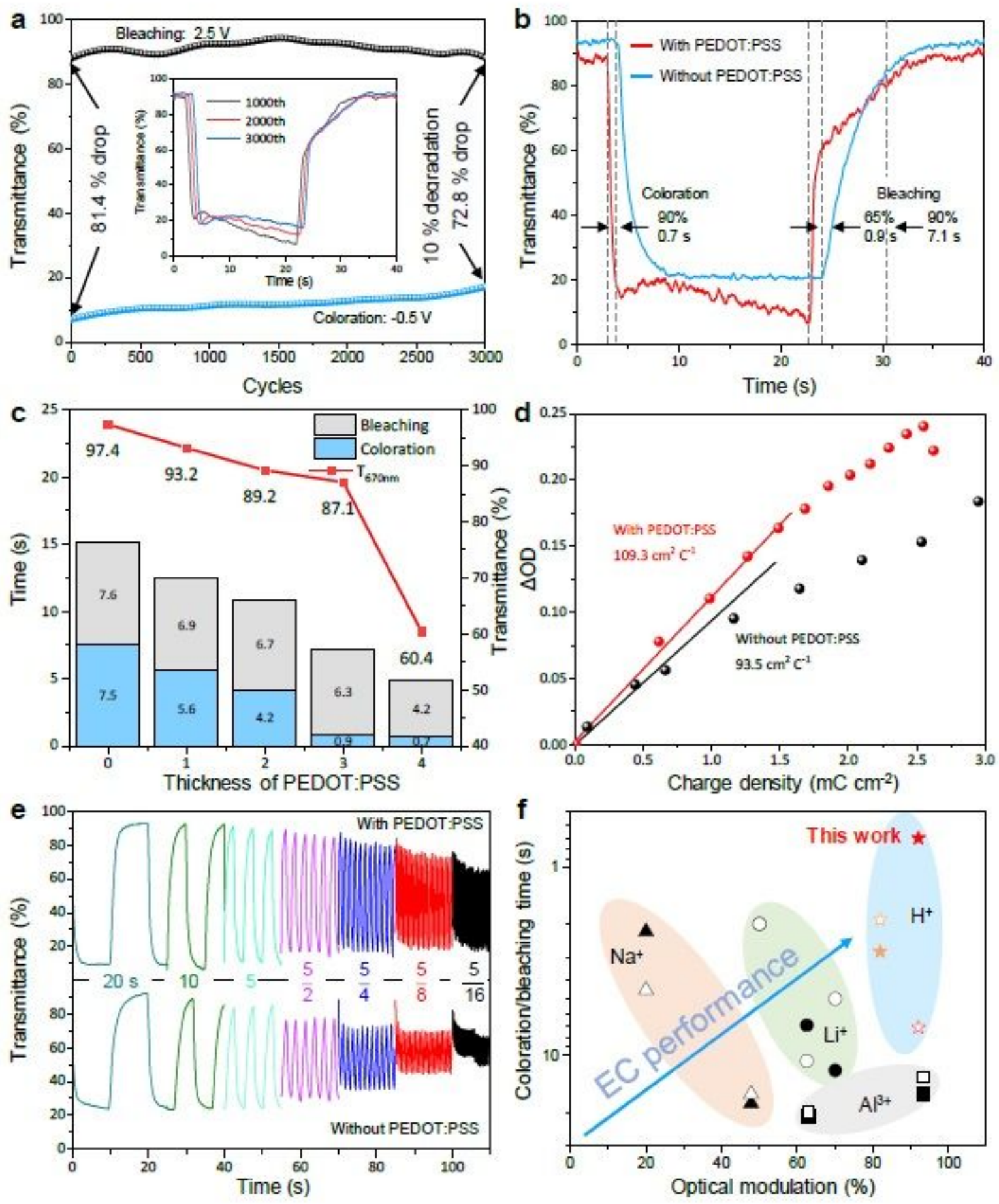

\section{Figure 3}

Performance of the tandem-structure ECD. (a) Real-time transmittance spectra of ECD at $670 \mathrm{~nm}$ under -0.5 and $2.5 \mathrm{~V}$ voltage, respectively. The inset is the transmittance spectra of the 1000th, 2000th and 3000th cycles; (b) Measurement of switching times from the transmittance spectra; (c) Relationship of the switching times and transmittance (at $670 \mathrm{~nm}$ ) with the thicknesses of the PEDOT:PSS layer. Here, the thickness was controlled by the times of spin coating. (d) Changes in optical density (at $670 \mathrm{~nm}$ ) with respect to injected charge density; (e) Response of transmittance to decreasing voltage pulse width from 
20 to 5/16 seconds; (f) Switching time and optical modulation of WO3-based ECDs with various insertion ions.
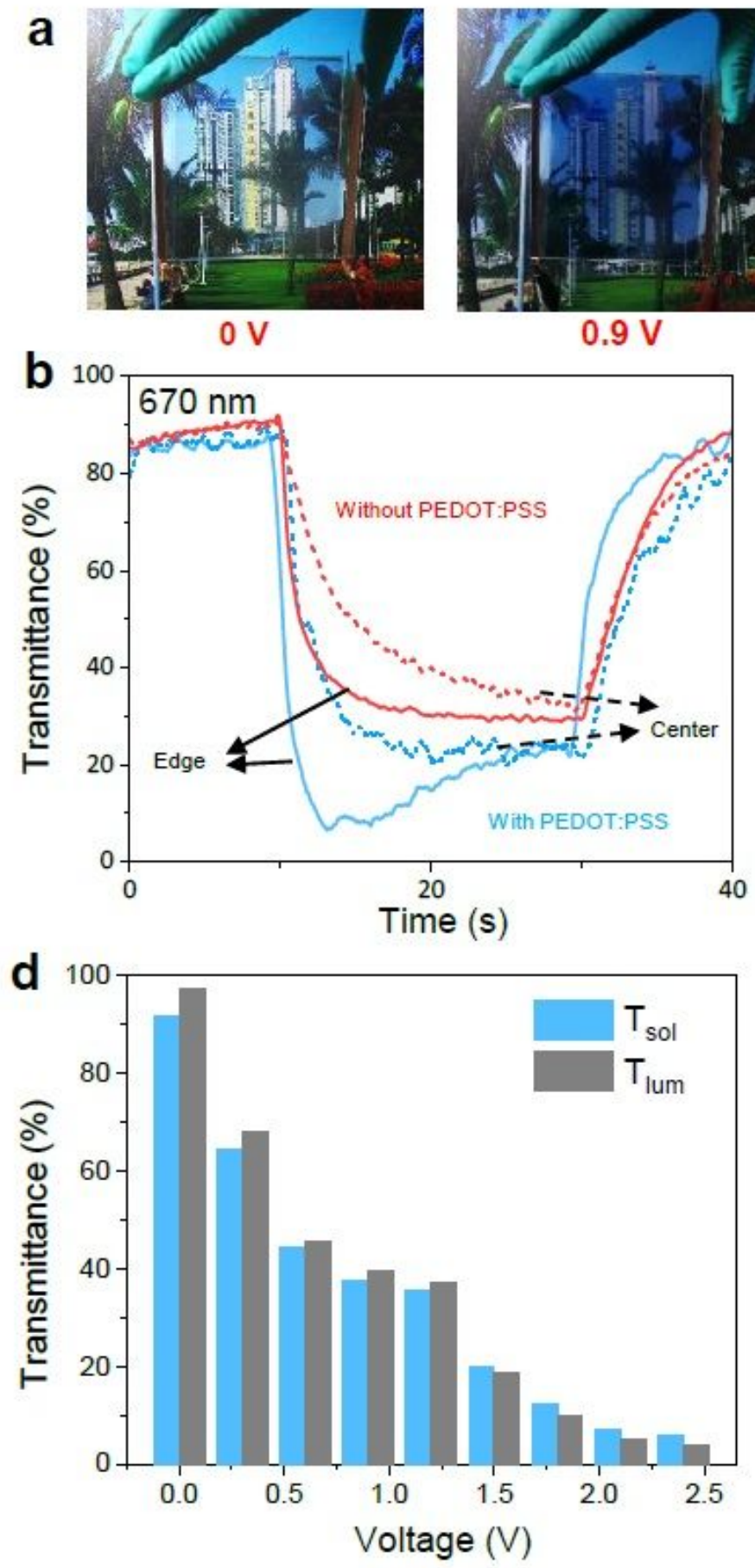

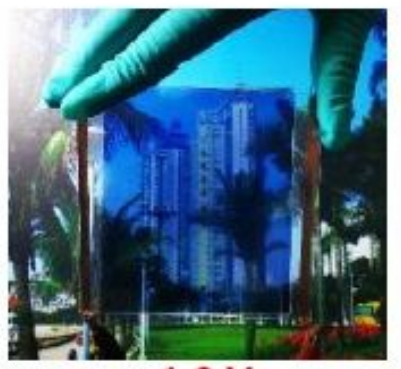

$1.2 \mathrm{~V}$

C

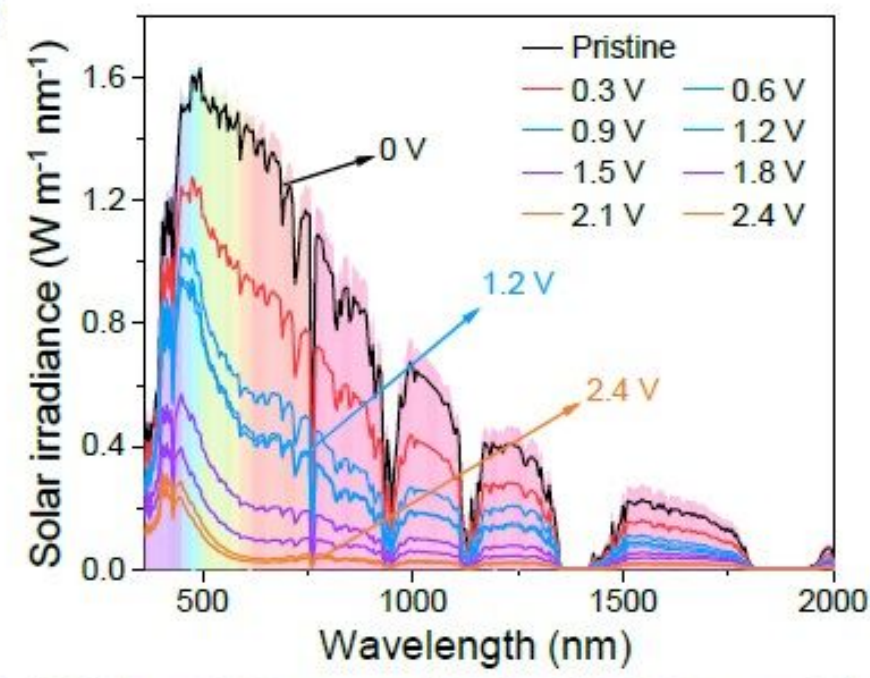

e

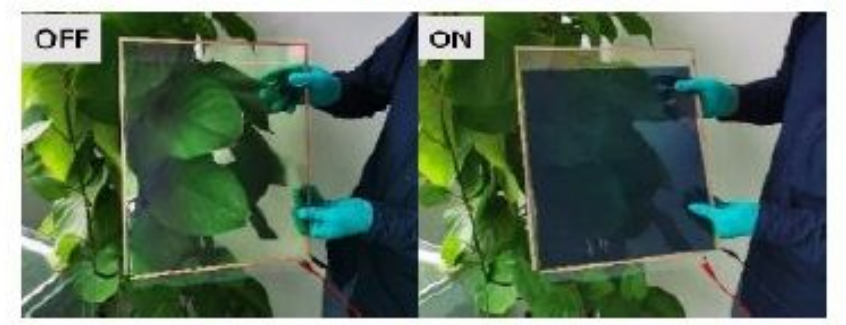

f

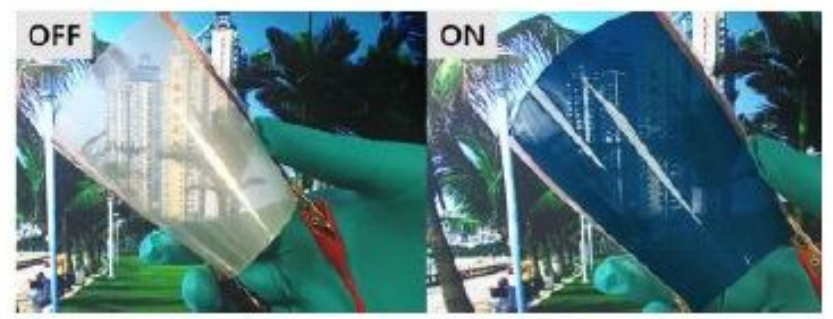

\section{Figure 4}

Scale-up of the tandem-structure ECD. (a) Photographs of a $10 \times 10 \mathrm{~cm} 2$ window under $0 \mathrm{~V}, 0.5 \mathrm{~V}, 1.5 \mathrm{~V}$ and $2.0 \mathrm{~V}$; (b) Transmittance at $670 \mathrm{~nm}$ at the edge (blue) and the center (red) of the window during switching at $2 \mathrm{~V}$ for $20 \mathrm{~s}$ followed by $-1 \mathrm{~V}$. For comparison, the results from a device of the same size but without the PEDOT:PSS layer are also shown; (c) Solar irradiance spectra converted from the measured transmittance spectra of the device; (d) Transmittance vs. voltage results for the all-solid-state EC device 
(blue bar for solar modulation ability and black bar for luminous transmittance). (e) Photographs of a $30 \diamond 40 \mathrm{~cm} 2 \mathrm{ECD}$; (f) Photographs of a flexible ECD.

\section{Supplementary Files}

This is a list of supplementary files associated with this preprint. Click to download.

- Supplementallnformation.pdf 